\title{
A Uni-Axial Nano-Displacement Micro-Tensile Test of Individual Constituents from Bulk Material
}

\author{
C. Du ${ }^{1}$ • J.P.M. Hoefnagels ${ }^{1}$ • L.I.J.C. Bergers ${ }^{1}$ • M.G.D. Geers ${ }^{1}$
}

Received: 4 August 2016 / Accepted: 22 May 2017 / Published online: 13 June 2017

(C) The Author(s) 2017. This article is an open access publication

\begin{abstract}
For both single-phase and multiphase metallic materials, it is necessary to understand the mechanical behavior on the grain-size scale in detail to get information that is not obtainable from macro-scale mechanical characterizations. This paper presents a methodology for uniaxial tensile testing of micro-specimens isolated from a bulk material. The proposed concept of multiple parallel micro-tensile specimens at the tip of a macro-sized wedge reduces the alignment work and offers an easy way for specimen handling. The selection of site-specific specimens is based on detailed microstructural and crystallographic characterization. Three kinds of representative specimens are presented to illustrate the wide range of application of the methodology for a variety of materials. Accurate, reproducible measurement of force $(2.5 \mu \mathrm{N}$ resolution) and displacement ( $\sim 10 \mathrm{~nm}$ resolution) is demonstrated, while accurate alignment (in-plane rotational and out-of-plane tilt misalignment of $<0.2^{\circ}$ ) limits the stress due to bending to $<0.2 \%$ of the imposed uni-axial stress. Combined with detailed material characterization on both sides of the microspecimens, this method yields detailed insights into the micro-mechanics of bulk materials which is hard to obtain from traditional macro-mechanical tests.
\end{abstract}

Keywords Micro-tensile test · Single-constituent testing · In-situ microscopy $\cdot$ Crystal orientation mapping $\cdot$ Site-specific specimen preparation

J.P.M. Hoefnagels

j.p.m.hoefnagels@tue.nl

1 Department of Mechanical Engineering, Eindhoven University of Technology, P.O. Box 513, 5600 MB Eindhoven, The Netherlands

\section{Introduction}

Multiphase materials are increasingly used in industry because the combination of different phases can yield a material with mechanical properties that exceed those of the individual phases, as e.g. typical for steels. Examples of multiphase steels include the advanced high strength steels, such as dual-phase (DP) steel, transformation-induced plasticity steel and twinning induced plasticity steel, all consisting of more than one phases such as ferrite, martensite, bainite and possibly (retained) austenite. The presence of multiple phases improves the overall mechanical response of materials for applications where traditionally conflicting properties are required, for example, high strength combined with good formability. In order to understand the contribution of each phase, one would like to measure its mechanical properties with a method that is able to isolate the neighboring phases and interfaces. It is generally too difficult to obtain the three-dimensional stress state locally in individual phases from a macro-specimen, which prevents detailed micro-scale analyses. Furthermore, the phases often influence each other during the fabrication process or even during storage and usage for some materials, which makes that the micro-mechanical behavior of the constituents different from that of a bulk material made of a single phase. Let us consider DP steel as an example. The martensite and ferrite phases influence each other in terms of their element distribution, phase volume fraction, and their mechanical behaviors are coupled [1]. For instance, carbon in the super saturated martensite diffuses towards the surrounding ferrite grains, which is one of the reasons why the ferrite grains close to martensite are stronger than those which do not share a boundary with martensite and thus have a lower carbon content. Therefore, the measured mechanical behavior of a macroscale specimen of a single phase material does not directly apply to the corresponding phase in the composite, which 
prevents accurate model predictions of the composite's mechanics. It would therefore be highly desirable to be able to perform a well-defined mechanical test on an individual constituent that is directly extracted from its multiphase surrounding.

Also for single-phase materials, a uni-axial tensile test on single grains is desirable. As each grain has different crystal orientations, the mechanics of single grains and grain boundaries should be understood to predict the overall material behavior. Macro-sized single-crystal specimens can be fabricated by enlarging the grains using heat treatments, e.g. [2]. However, for engineering materials, the high alloy content typically prevents the growth of huge crystals at low cost. Moreover, even for a single-phase material it is often incorrect to directly apply the conclusions of a macro-scale single crystal test to micron-sized grains in the associated engineering materials. Micro-sized grains show discrete mechanical behavior due to a limited number of dislocations, precipitates, etc. Moreover, so-called 'size effects' in mechanical behavior of single-phase materials have triggered intense research in the last decades [3, 4], and the extrinsic size effect, i.e. due to the specimen size, should not be neglected when projecting macro-scale tests to the micro-scale. For more complex single phase material like bainite and martensite, which has a fundamental structure unit, the so called lath, with the thickness of sub-micrometers, it is critical to understand the material behavior well at the 'lath scale'. The internal (packet- / block- / subblock-) boundaries, between groups of laths, as found in martensite and bainite, are critical ingredients to improve the mechanical properties of advanced high strength steels [5]. However, their roles on the mechanics have so far only been studied indirectly through macro-scale specimens $[6,7]$, or from micro-specimens subjected to a complex loading state (e.g. bending test $[8,9])$. Therefore, mechanical tests with a simple stress state at the micron scale are essential to understand the complex mechanics of simple single-phase materials, complex single-phase materials (e.g., bainite and martensite) and their size effects, as well as composite and multiphase materials as stated above.

As argued above, it is important to test the mechanical properties of individual phases or micro-sized grains. Therefore, a dedicated micro-mechanical test methodology is developed, which should satisfy the following requirements: (1) it should be with capacity to test individual phases; (2) the size of the micro-specimens should be smaller than the grains of individual phases; (3) specimen preparation methods should give a high shape accuracy of the specimens on the corresponding scale; (4) the handling of these micro-sized specimens, as an integral part of the methodology, should be relatively simple, since that transportation and mounting of the micro-sized specimens without pre-loading them is a well-known challenge; (5) it is important that the method allows for site-specific specimen fabrication based on detailed micro-structural characterization over a large material area (e.g. local crystal orientation/chemical composition), which in turn will also assist the analysis of the mechanical behavior afterwards; (6) the applied loading and deformation should be measured with high sensitivity and accuracy; (7) in-situ testing should be feasible in order to record the complete deformation process of the specimens.

Among the existing methods of micro-mechanical testing, static tests like micro-bending tests $[8,9]$ and micro-torsion [10] as well as micro- and nanoindentation [11, 12] are often used. However, for these tests, the local stress state (in the gauge section) is hard to obtain due to the interaction of the (highly) inhomogeneous loading state with the microstructure which is also inhomogeneous at the same length scale. This makes it difficult to derive exact values of the mechanical properties. In contrast, similar to testing at the macro scale, uni-axial tension and compression are mechanical tests with straight-forward loading conditions. The micro-pillar compression test is widely applied, for example, Jirkova et al. investigated the effect of retained austenite on micro- and macro-mechanical properties of quenching and partitioning steels [13], while Kheradmand et al. studied the role of grain boundary in plastic deformation using micro-pillar compression test [14]. However, compression and tensile tests cannot replace each other, because the small-scale mechanics is never exactly the same, for instance: (1) the onset of localization and the initiation and evolution of damage and fracture is completely different, and (2) for BCC metals, the so-called non-Schmidt effect [15] causes the plastic behavior under tension and compression to be asymmetric. Therefore, a well-defined micro-tension test would be a welcome addition to the many micropillar compression tests in the literature and to yield deeper insight into the micro-mechanical mechanisms underlying the macroscopic behavior.

The aim of this study is to establish a highly-sensitive and reproducible methodology for uniaxial micro-tensile testing of single components extracted from multiphase materials and testing of individual grains of single-phase materials. The paper discusses the details of the methodology designed for testing of micro-tensile specimens from bulk materials, which includes specimen preparation, specimen alignment and the procedures for uniaxial tensile testing. Tests on single-grain specimens made from interstitial free (IF) steel and micron-sized martensite specimens with a selected orientation relative to the loading direction will serve to demonstrate the capability for micro-tensile testing of single-phase materials. Tests on ferrite specimens from DP steel will demonstrate the capability for testing of a single phase extracted from a multiphase material. 


\section{Experiments}

\section{Specimen Fabrication}

The design of the experiment needs careful considerations due to the miniature size of the specimens. Starting from the bulk material, first a wedge is prepared with a tip that ends in a straight knife edge. Next, a detailed analysis of the microstructure and crystallographic orientation is performed on both the front side and the backside of the wedge. Finally, micro tensile specimens are fabricated from the wedge tip in such a way that they remain attached to the wedge, making them easy to handle. Focused ion beam (FIB) is chosen to fabricate the microsize tensile specimens because of its fabrication accuracy. Many FIB fabricated micro-mechanical testing specimens are available in the literature, examples are found in Refs. $[3,4,8,9,16-19]$. Care should be taken that the wedge tip is thin enough to enable FIB milling of the specimens, with a thickness that is smaller than the length scale of the microstructure, such that a double-side analysis of the wedge tip is representative for the final micro-tensile specimens to be fabricated from the wedge. For instance, when testing single grains, the double-side measurement ensures that each specimen consists of a single grain. For the specimens with internal boundaries, the double-side measurement gives detailed information of the number and position of boundaries, the boundary orientation (inclination) in 3D and crystallographic information of the grains.

Preparation of the wedge is realized by grinding a piece of bulk material under an angle into a wedge shape. The original bulk sheet material with thickness $t$ is cut into small chip-sized windows with a dimension of $10 \times 9 \times t$ in $\mathrm{mm}(t=1.0 \mathrm{~mm}$ for all tests shown here). The back side of the piece is mechanically ground and polished. Subsequently, the front side is ground and polished under an angle, by gluing the bottom to a specimen holder of a semi-automatic grinding/polishing machine ('Target machine' from Struers), which allows the front side to be tilted at the desired angle with respect to the grinding/polishing disk. After both sides have been polished, a wedge has been produced with a razor sharp tip edge equal to the tilt angle on one side and an end thickness of roughly $1 / 2 t$ on the other side. Even though the grinding and polishing is performed carefully with many fine polishing steps (ending with a $10 \mathrm{~min}$ OPS polish with $40 \mathrm{~nm}$ silica particles), it is obvious that micron-scale mechanical deformation will always be introduced to the wedge tip, as can easily be seen from the waviness of the tip in Fig. 1(a). This means that the wedge tip material has undergone hardening and perhaps even damage. It is therefore essential to remove the deformation layer of the wedge with a method, which does not introduce (new) mechanical deformation. Therefore, electrochemical polishing is subsequently applied. A schematic illustration of the electrochemical polishing process is shown by the insert of
Fig. 1(b). The voltage is $30 \mathrm{~V}$ and the flow rate is $15-20$ and the polishing time is ranged from $15 \mathrm{~s}$ to $30 \mathrm{~s}$ using the LectroPol 5 from Struers with Electrolyte A2. These parameters were carefully optimized to obtain a perfect finish, as shown for a DP steel wedge in Fig. 1(b). The wedge tip is straight and smooth, i.e. the waviness has been removed, which suggests that the deformed layer by grinding showed in Fig. 1 has been completely removed. Moreover, the phase/ grain boundaries of this DP steel are continuous from one side to the opposite side, which means that there is only one grain in the thickness direction. Note that the electrochemical polishing process should not be too long, because it rounds off the tip angle, which leads to a (much) longer FIB time in the subsequent steps. To verify that after electrochemical polishing the wedge is sufficiently deformation free, an EBSD scan of both sides of the edge region of the wedge has been carried out. The resulting inverse pole figure map, image quality map, and kernel average misorientation map are shown in Fig. 2(b-d). The gauge parts of the future microtensile specimens are located between the white dashed lines, where it can be seen that the large (ferrite) grains, which would most easily reveal mechanical deformation due to grinding and polishing, have a common inverse pole figure color with a kernel average misorientation that remains below $\sim 1^{\circ}$, indicating that the crystals are relatively clean. Although not done here, the initial dislocation density can be quantified using transmission electron microscopy, or the initial dislocation density can be further reduced by adding an annealing step of the wedge in high vacuum (for cases where alteration from the original, as-received microstructure is not a problem).

Note that the image quality map in Fig. 2(c) may appear to show deep grain boundary grooves due to the electrochemical polishing, however, this is misleading. From detailed analysis of edge-on images similar to Fig. 1(b), it is shown that the apparent grain boundary grooves are actually rounded-off steps with a typically height of a few tenth of nanometers, i.e. approximately $1 \%$ of the specimen thickness. Therefore, these smooth steps should not give rise to large stress concentrations.

At the location of the gauge section of the specimens (dashed white line in Fig. 2) the wedge tip is thin enough, i.e. with a thickness of less than $10 \mu \mathrm{m}$, which allows to mill specimens by FIB. This concept of a thin macro-wedge from which multiple parallel micro-compression specimens are milled with FIB is similar to the method of micro-wedge used in [20], the $9 \mathrm{~mm}$-long wedge tip allows to have many parallel micro-tensile specimens, which are free standing but are connected to the wedge at their base (as shown by the blue specimen contour in Fig. 2(a)). The difference to the method in [20] is that an extra electro-chemical polishing process is introduced to remove the deformation caused by mechanical grinding and polishing step. This macro-wedge permits easy transportation, mounting and demounting of the micro-tensile 

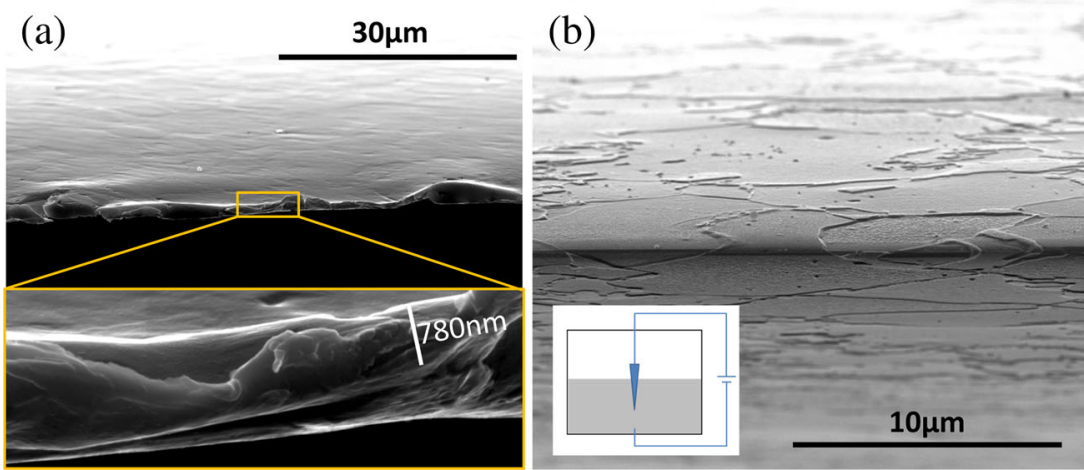

Fig. 1 (a) Scanning electron microscope (SEM) secondary electron (SE) image of front view of the wedge tip after mechanical polishing, with obvious mechanical deformation at the edge tip. (b) SEM SE image of a DP steel wedge after electrochemical polishing, observed from the top of the wedge. The tip of the wedge is sharp in the center, across which clear grain/phase boundaries running from the front side to the back side are visible. The insert shows a schematic illustration of the adopted electrochemical polishing process with wedge tip dipped in electrolyte

specimens since one only needs to handle the wedge, as opposed to stand-alone micro-tensile specimens that requires dedicated effort to assemble a micro-sized specimen into the grippers using micromanipulators (e.g. in [21]). In addition, compared to a single free-standing specimen fabricated on top of a needle specimen, where only one micro-tensile specimen can be fabricated [22], the current wedge method saves time for mounting and aligning of the specimens in the tensile stage since all micro-tensile specimens are parallel as they are milled with FIB in a single process.
If a specific region needs to be selected, the wedge concept is especially suited as it allows for high resolution microstructural characterization from both sides, e.g., with EBSD as shown in Fig. 2. The selection can be morphological (e.g. a certain phase, grain boundary regions, or inter-grain regions in a multiphase material), or crystallographic (e.g. a certain region with a specific orientation or a certain type of grain boundary). In fact, due to the FIB procedure used, as discussed below, the backside of the micro-specimen is part of the backside of the wedge, i.e. it is not touched by FIB.

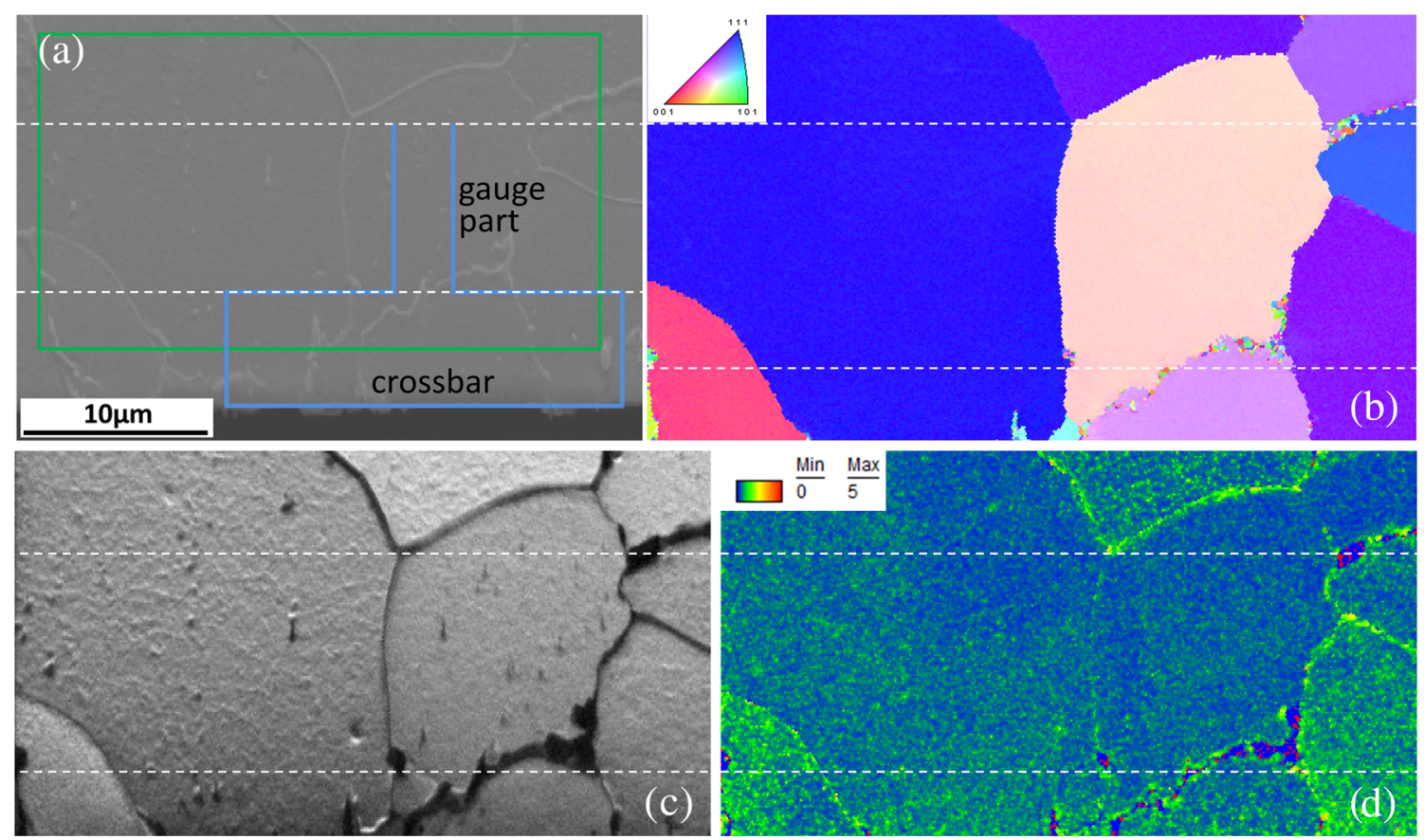

Fig. 2 EBSD measurement of the wedge tip fabricated from a DP600 steel sheet as an example. (a) SEM SE picture of the edge part, with internal grain/ phase boundaries. The green frame indicates the area that is measured with EBSD and shown in subplots (b-d). For reference, the blue frame represents the contour of a T-shaped micro-tensile specimen (consisting of a rectangular gauge section and crossbar for load application), while the area between the white dashed lines is the region where the gauge part of the micro-tensile specimens will be located, as also indicated in subplot (b-d). (b) An inverse pole figure map of the scanned area; the uniform color within each grain indicates a common orientation. (c) the EBSD image quality map of the same area, where the grain/phase boundaries are clearly visible. A high image quality is obtained in the ferrite grain; the black regions are martensite. (d) Kernel average misorientation map of the scanned area, which shows very small misorientation in the ferrite grains; this confirms that the wedge is free from mechanical deformation, since the large and relatively soft ferrite grains would be deformed first 
Therefore, the morphology of the grains at this wedge side, revealed by the electrochemical polishing, can be imaged with SEM in SE mode. Hence, a millimeter-sized area can be scanned quickly for selecting interesting micro-specimen fabrication sites, e.g., with a specific grain boundary orientation. Of course, additional EBSD analyses are needed if selection should occur on the basis of phase orientations. An example of this region selection for martensite with a preferred orientation based on EBSD measurements is shown below.

It is well known that the gallium ion implanting during FIB milling can introduce surface dislocation loops and precipitates and even instigate amorphous layers, which may change the mechanical property of the specimen in a thin surface layer [3, 23-31]. Careful side-by-side studies of compression tests on pillars prepared both with and without FIB consistently show a distinct change in mechanical behavior, see e.g. [30, 31]. Unfortunately, it was also not found possible to remove the FIB-induced damage layer by a second electrochemical polishing step, because the initiation of electrochemical polishing is intrinsically unstable, resulting in pitting, whereas the ultra-high quality surface finish of Fig. 1(b) is only achieved after removal of the first $\sim 10 \mu \mathrm{m}$. Note, however, that the largest influence of FIB-induced damage on the mechanics was found in "size effects" studies on specimens with very small dimensions $[3,27]$. Therefore, we here adopt (much) larger specimen dimensions (i.e. a thickness and width of 2.5 and $3 \mu \mathrm{m}$ ), for which a smaller detrimental effect would be expected. In addition, although the FIB-induced damage layer can never be eliminated completely, its thickness and effect on the specimen's mechanics can be minimized by lowering the beam energy, beam current, and landing angle [27].

Given the considerations above, the milling process was set up to minimize the thickness and mechanical influence of the FIB-induced damage layer. The milling process is shown in Fig. 3 schematically. The micro-tensile bars are fabricated with a dual beam SEM-FIB (FEI Quanta 3D FEG), using an ion acceleration voltage of $30 \mathrm{kV}$ progressing from a larger beam current for coarse milling to a finer beam current for a final surface finish. First the specimen is placed such that the back plane of the wedge is perpendicular to the ion beam and a T-shaped micro-specimen is milled with a beam current of $3 \mathrm{nA}$ at the wedge tip. At this point the thickness of the gauge part of T-shaped micro-specimen still decreases towards the wedge tip. Secondly, the back plane of the wedge is aligned parallel to the ion beam, see Fig. 3(b), and using an ion beam current of $1 \mathrm{nA}$ a small layer is removed from the specimen top plane, thereby making the gauge section plan-parallel and removing the prior $3 \mathrm{nA}$ FIB-induced damage. Furthermore, a $0.3 \mathrm{nA}$ surface cleaning is applied to remove the $1 \mathrm{nA} \mathrm{FIB-}$ induced damage and provide an accurate final shape. In the third step, the back plane is rotated perpendicular to the ion beam, as in step one. The side planes of the micro-specimens are cleaned with beam current $0.3 \mathrm{nA}$ to obtain a high shape accuracy and surface quality, and to remove most of the FIBinduced damage. This is confirmed by the fact that the EBSD image quality in the gauge section after FIB milling (Figs. 12 and 13) is very high, as high as the EBSD image quality of the wedge before FIB milling (Fig. 2). Therefore, the FIB-induced damage layer must be thinner than the so-called "EBSD depth resolution" (i.e. the EBSD interaction volume resulting from only the coherent backscattered electrons of the primary beam), which for our case is estimated to be thinner than $20 \mathrm{~nm}$ according to [32]. Hence, considering the specimen thickness and width is 2.5 and $3 \mu \mathrm{m}$, the influence of the FIB milled surface layer on the specimen's mechanics is estimated to be negligible. Note that the backside has not been exposed to FIB at all. Special care is taken to ensure that the micro-tensile specimens on the same wedge have parallel axes. This means that during the fabrication of all specimens, no rotation of the wedge is made between micro-specimens during the first step and the third step. In the second step, the tilt angle of the wedge is also kept the same for all micro-tensile specimens. Note that a possible thickness variation along the specimen length is of concern. It is known from literature that parallel ion beam milling of a specimen always results in a slight tapering of the specimen along its length, on the order of $\sim 2^{\circ}$ in this case, similar to [33, 34]. Therefore, the tilt angle of the wedge with respect to the ion beam was simply adjusted to correct for this effect, yielding a maximum thickness variation over the complete gauge section of less than $1 \%$ of the thickness, as verified from side-view SEM images from both sides (not shown).

To analyze the loading that is applied by the two-pin gripper on the specimen, a linear elastic, plane stress FEM model of the specimen geometry and displacement loading condition has been developed with a converged mesh of eight-node biquadratic elements with eight-point Gaussian integration with a mesh refinement at the sharp inner corners of the specimen geometry (connected with linear tying constraints), as shown in Fig. 4(a). The elastic parameters are chosen to correspond to steel $(\mathrm{E}=210 \mathrm{GPa} ; \nu=0.3)$, while the magnitude of the applied displacement (of $42 \mathrm{~nm}$ ) by the two-pin gripper has been chosen such that the axial stress in the gauge section, $\sigma_{\mathrm{yy}}$, equals the martensite yield stress, here assumed to be $800 \mathrm{MPa}$. An inner corner radius of $50 \mathrm{~nm}$ was chosen as a compromise between a shorter gauge section with uniform cross section for larger radius and a higher stress concentration at the corners for smaller radius. A minimum radius of $50 \mathrm{~nm}$ was also observed in SEM and has, therefore, been included in the FEM model, see insert in Fig. 4(a). As can be seen from the displacement, stress, and strain fields in $\mathrm{y}$ - and $\mathrm{x}$-direction (Fig. 4(b,c,d)), a homogeneous uni-axial tensile state is achieved over almost the complete central part of the gauge section. This is in agreement with the general experimental observations that most plasticity events (e.g. all slip bands and localizations in the single-crystal specimens discussed in 


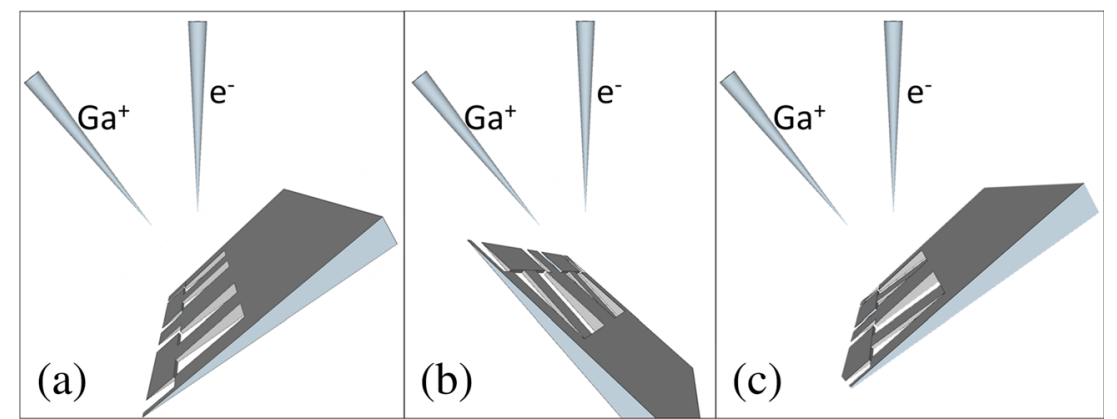

Fig. 3 Schematic illustration of the FIB milling process of the tensile bars. The tilt angle of the ion beam with the bottom side of the wedge is $90^{\circ}, 0^{\circ}$, and $90^{\circ}$ for, respectively, step (a), (b) and (c): In the first step (a), the backside of the wedge is aligned to be perpendicular to the ion beam for a coarse milling of T-shaped specimens. Second (b), the wedge is aligned with the backside parallel to the ion beam axis, after which the top side of the micro-tensile specimens is milled under shallow angle to obtain a constant specimen thickness. Finally, in (c), the wedge is aligned again with the backside perpendicular to the ion beam for the fine milling of the specimen side which yields better surface finishing quality and higher shape accuracy

Fig. 11 below) occur somewhere in the region of homogeneous uni-axial tensile state, and thus away from the gauge ends where the deformation is more constrained. Therefore, this confirms that the developed micro-tensile methodology indeed applies a uni-axial tensile state to the gauge section cut from the bulk material.

\section{Experimental Setup}

The tensile test is conducted with a recently built microtensile stage that is equipped with accurate force and displacement measurement techniques and allows for precise specimen alignment. The micro-tensile stage is composed of two modules, as is shown in Fig. $5(\mathrm{a}, \mathrm{b}, \mathrm{c})$. The specimen is mounted on the left module indicated with 'wedge mounting and rotation' in figure Fig. 5(b), which can be rotated in $x-y$ plane about the z-axis in a range of $\pm 0.7^{\circ}$ and tilted in the $\mathrm{x}-\mathrm{z}$ plane about the $\mathrm{y}$-axis in a range of $-1.9^{\circ}$ to $0.5^{\circ}$. Due to the final electro-chemical polishing step of the wedge, the backside of the wedge shows a minor curvature over the first $\sim 100 \mu \mathrm{m}$ starting from the tip inwards, which causes the specimen back side
Fig. 4 (a) The specimen geometry $\left(\mathrm{l} \times \mathrm{w} \times \mathrm{h}=9 \times 2.5 \times 2 \mu \mathrm{m}^{3}\right)$, with the applied displacement boundary condition of two-pin gripper on the top-side of the cross-bar as shown by the two arrays of pink arrows, and the ultra-fine mesh with $2.5 \mathrm{~nm}$ element size in the four inner corners with a radius of $50 \mathrm{~nm}$. $(\mathbf{b}, \mathbf{c}, \mathbf{d})$ Because the problem is mirror symmetric, the left and right side of each image shows a different field: (b) the displacement in $\mathrm{y}$ - and $\mathrm{x}$-direction, $\mathrm{u}_{\mathrm{y}}$ and $\mathrm{u}_{\mathrm{x}},(\mathbf{c})$ the stress in $\mathrm{y}$ - and $\mathrm{x}$-direction, $\sigma_{\mathrm{yy}}$ and $\sigma_{\mathrm{xx}}$, (d) the strain in $\mathrm{y}$ - and x-direction, $\varepsilon_{\mathrm{yy}}$ and $\varepsilon_{\mathrm{xx}}$

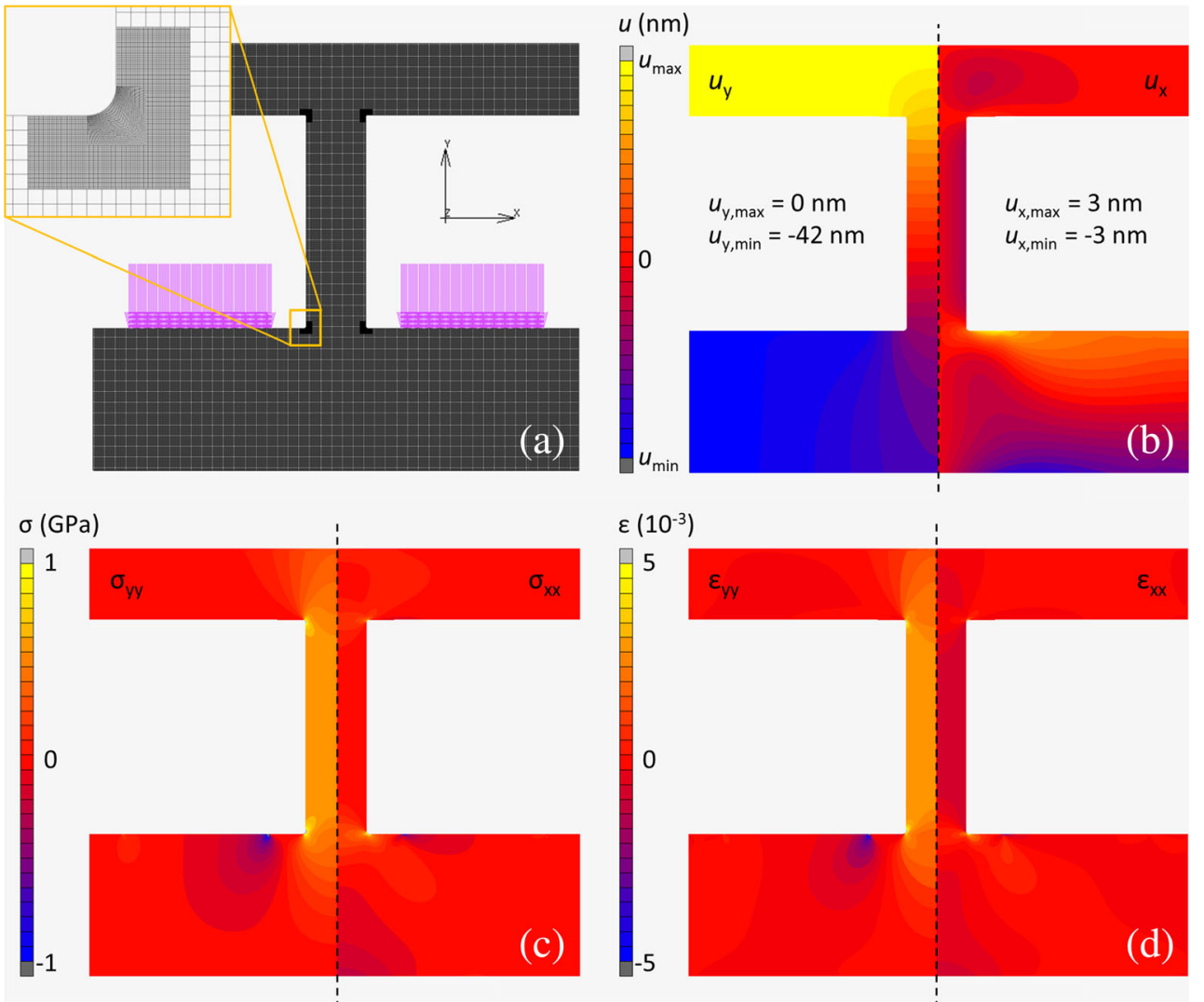




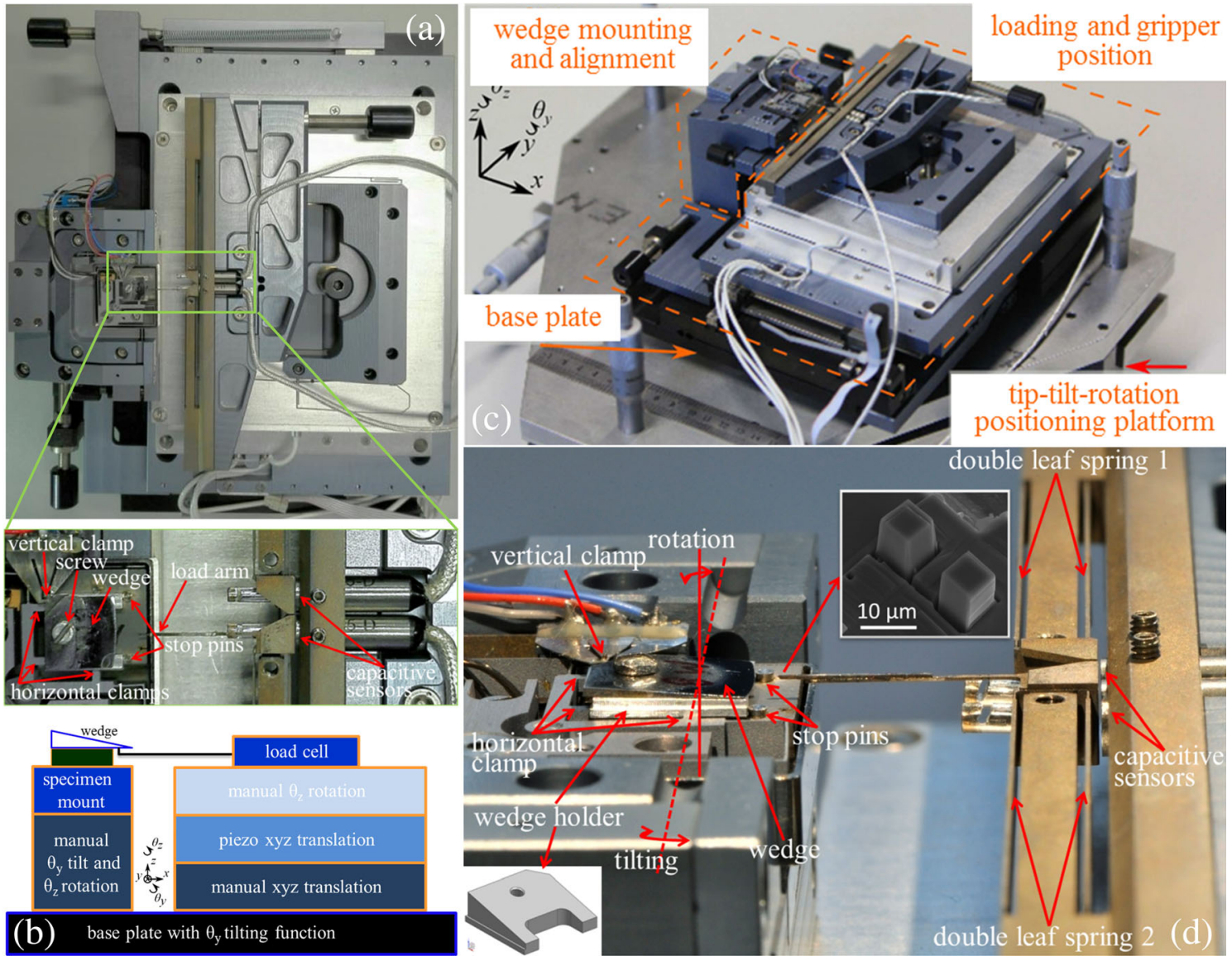

Fig. 5 (a) A top view of the micro-tensile stage, with a zoom of the specimen mounting mechanism and the loading arm part of the load cell. (b) Schematic drawing of the micro-forced tensile stage from the size view, which clearly shows the specimen mount part on the left and the specimen loading part on the right. (c) the micro-tensile stage mounted on the top of a tilt-rotation positioning stage. (d) a closer view of the specimen mounting configuration and the double leaf spring load cell and double leaf spring reference cell. The top insert shows the two-teeth loading gripper and the bottom insert a sketch of the tapered wedge holder

to be tilted a few degrees with respect to the wedge end. To tilt back all micro-specimens in a wedge correctly, a simple pre-tilted wedge holder (see insert in Fig. 5(d) has been designed, using a pre-tilt angle that is measured separately for each wedge using surface profilometer. The wedge is fixed by a small screw (see the zoom of Fig. 5(a)) onto this wedge holder (bottom insert of Fig. 5(d)), which is then mounted on the specimenmounting module by a set of horizontal clamps, which push the holder slightly downwards against three stop pins and a vertical clamp, as shown in Fig. 5(a,d). This ensures a stable specimen mounting throughout the testing process.

The main component of the right module is a piezo-block which can be positioned coarsely in $\mathrm{x}, \mathrm{y}$ and $\mathrm{z}$ directions by manual screws, while a fine movement with $7 \mathrm{~nm}$ precision over a $200 \mu \mathrm{m}$ range that is realized using the $\mathrm{x}, \mathrm{y}, \mathrm{z}$ piezoblock activation (MCL NanoT225). The left side of the piezo block contains a custom load cell, which consists of one double leaf spring mechanism that measures the force on the specimen and a second double leaf spring mechanism to correct for systematic (e.g. temperature) influences. The first double leaf spring mechanism holds a long free-standing loading arm. At the left end of the loading arm, a gripper is located, which has two micron-sized rectangular teeth milled with FIB pointing upwards, as shown in the top insert in Fig. 5(d). These two teeth are used to load a micro-specimen by pulling at its crossbar. The high accuracy of the force measurement will be validated below. The tensile test is carried out under a surface profilometer (Bruker NPFLEX) on a vibration-isolated table to minimize external vibrations. In addition, the tensile stage is also designed for in-situ testing inside a SEM. More design details of the micro-tensile stage can be found in [35].

\section{Specimen Alignment and Testing}

After mounting the wedge on the micro-tensile stage, accurate specimen alignment with respect to the loading direction is required to avoid complex loading caused by bending [21, 35]. Following criteria by Bergers et aI. [35], for example, for a micro-tensile specimen gauge dimension of $9 \times 3 \times 2.5$ $\mu \mathrm{m}^{3}$, the in-plane rotation misalignment between the specimen axis and the loading axis has to be smaller than $11 \mathrm{mrad}$ 
$\left(0.68^{\circ}\right)$, and the out-of-plane tilt misalignment may not exceed $10 \mathrm{mrad}\left(0.57^{\circ}\right)$ to limit bending stress to $0.5 \%$ of the imposed uni-axial stress.

The specimen alignment involves two steps, the out-ofplane alignment (tilt) and the in-plane (rotation) alignment. The tilt alignment, which involves the out-of-plane alignment of the load axis to the specimen axis, is shown in Fig. 6(a-c). Because the load axis is not necessarily exactly parallel to the top surface of the loading arm, the tilt angle of the load axis is measured by tracking the 3D displacement of the loading arm using Digital Height Correlation (DHC), which is a recentlydeveloped form DIC to correlate surface height profiles instead of grey scale images [36, 37]. In this case, surface profiles of the region in the white frame of the loading arm are measured by optical profilometry at two positions at large horizontal distance from each other ( $200 \mu \mathrm{m}$ displacement), see Fig. 6(a,b). The three-dimensional displacement of the load axis, resulting from the DHC analysis, is then compared with the tilt angle of the micro-specimen, which is obtained by directly fitting a plane through a surface profile of the specimen gauge part, as indicated by the white frame in Fig. 6(c). The tilt misalignment is calculated and corrected by tilting the specimen mounting block toward the load axis. The procedure is repeated until the tilt misalignment is $0.2^{\circ}$ or less, which is well within the allowed range. Typically, one or two iterations suffice to reach this tilt accuracy.

Similarly, for the rotation alignment, the in-plane difference between the specimen axis and the load axis also needs to be corrected. The above-measured 3D load axis, obtained with $\mathrm{DHC}$, is also used to correct this inplane rotational misalignment with respect to the field of view. To this end, the specimen axis direction is obtained by detecting the edge of the specimen gauge via thresholding of the pixel intensity, as shown in Fig. 6(d). The two horizontal lines in the gauge part of the specimen are two edges of the specimen front surface, which are parallel to the specimen axis. The vector describing the specimen axis is obtained by averaging the directions of these two lines. The in-plane rotational misalignment between the load axis and specimen axis is then obtained and corrected by rotating the specimen axis towards the load axis. This process is again repeated until the tilt misalignment is $0.2^{\circ}$ or less, which is well within the allowed range. The combination of an in-plane rotational and out-of-plane tilt misalignment of $<0.2^{\circ}$ limits the stress due to bending to $<0.2 \%$ of the imposed uni-axial stress.

The micro-tensile test can now be executed. The gripper is moved from the bottom toward the plane of the micro-specimen, as shown in top-view optical images in Fig. 7(a,b), after which the gripper is moved toward the crossbar of the specimen for loading (Fig. 7(b,c)). The cross bar is now displaced to apply tension and Fig. 7(d) shows that two slip traces appear on the specimen surface.

\section{Force and Displacement Measurement and Stress-Strain Curve}

The small dimensions of the tensile specimens require an accurate force measurement. For a typical fracture stress of $600 \mathrm{MPa}$, the fracture force of the tensile specimen is $6 \mathrm{mN}$ for a specimen cross section of $10 \mu \mathrm{m}^{2}$. Therefore, a load cell is connected to the load arm with a force range $0-25 \mathrm{mN}$ and a precision of $10^{-4}$ of the full range, corresponding to a load resolution of $2.5 \mu \mathrm{N}$. The basic mechanism of the load cell is
Fig. 6 The specimen alignment procedure. (a,b) Loading axis out-of-plane (tilt) and in-plane (rotation) angle measurement by tracking the displacement of the white frame area in 3D. (c) Specimen loading axis out-ofplane (tilt) angle measurement by fitting the plane of the gauge part of the specimen in the white frame. (d) Specimen loading axis in-plane rotation angle measurement by edge detection
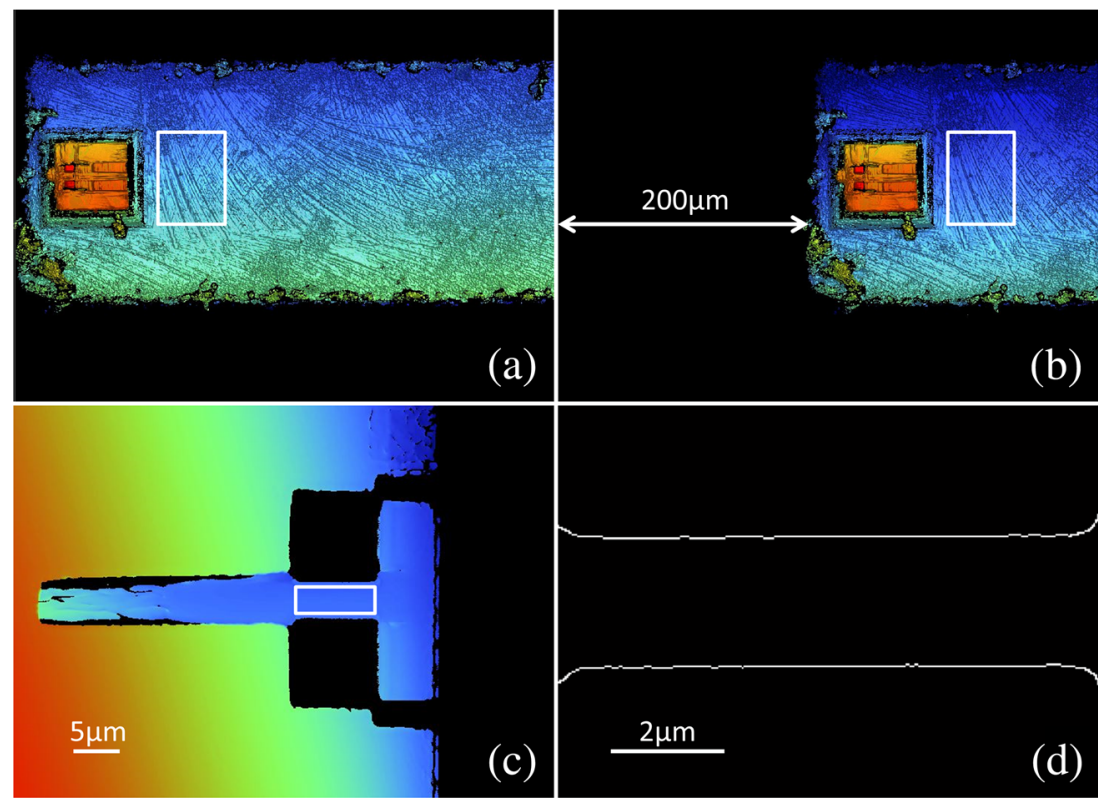


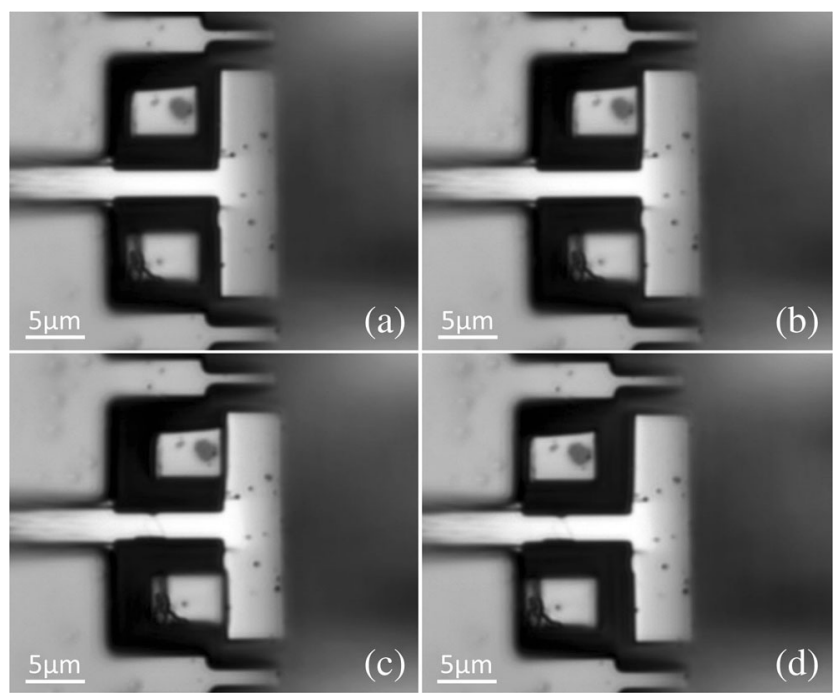

Fig. 7 Top view optical image series of the gripping process and tensile test of a $9 \mu \mathrm{m}$-long micro-tensile specimen. (a) The specimen is gripped from the bottom. (b) The gripper is moved toward the crossbar of the specimen at which the loading is started in (c) after the contact with the crossbar. (d) Slip traces appear on the surface of the specimen gauge part, after which the griper is reversed to interrupt loading, in order to avoid severe plastic deformation for a better identification of the slip systems. In case a complete stress-strain curve of the specimen is required, the loading continues until the specimen is fractured

that of the measurement of the deflection of a leaf spring with known elastic stiffness, as explained as follows.

As stated already, the gripper with its two teeth is fabricated at the tip of a (long) load arm, which is attached to a doubleleaf spring mechanism, which constrains all degrees of freedom of the gripper motion except for the forward/backward motion, see Fig. 5. The force measurement is realized by measuring the deflection under load of the double-leaf spring mechanism with a capacitive sensor that is mounted behind the load arm and aligned along the specimen/load axis (Probe C5-D, driver CPL-190 from LionSensor, see Fig. 5(b)), i.e. the capacitive sensor and the double-leaf spring are the two components of the load cell. The calibrated leaf spring stiffness is designed to yield the desired force range, as explained in Ref. [35]. For such minute forces, the force measurement is highly sensitive to background influences caused by thermal fluctuations, environmental vibrations, and tilt-induced deflections of the leaf spring. Therefore, to measure these background influences, a second identical double-leaf spring is designed in a mirrored configuration as part of the same load cell. The corrected force measurement is obtained by subtraction of the specimen force by the background force with a sampling rate of $10 \mathrm{~Hz}$; more details are given in [35]. The effectiveness of this corrected measurement can be seen at the left of Fig. 9(a) below, as the corrected noise in the force is small compared to the applied load to the micro-tensile specimen. The two double-leaf spring mechanisms has been fabricated by wire electrical discharge machining (EDM) of a TiAlV alloy with a high yield strain (and thus large maximum elastic deflection) and good manufacturing properties. The load arm end with a cube size of $50^{3} \mu^{3}$, which was produced by wire EDM from a $0.3 \mathrm{~mm}$-thick plate of TiAlV. Using FIB milling, the top of the cube has been further milled to shape the gripper with its two loading teeth, as shown in Fig. 5(b).

Similar to Ref. [38], the global displacement measurement is performed by extracting relative rigid body displacements of specimen regions by digital image correlation, see Fig. 8, although here a dedicated global digital image correlation (GDIC) algorithm [35, 37] has been employed for improved accuracy. Images are taken from the start of loading until the fracture of the specimen. The blue frames in Fig. 8 are correlated to obtain the rigid body motion during the test, caused by drift in the test setup. The red frame at the end of the specimen is tracked as well. Initially, the contrast at the edges between the material and the empty regions in the frames was used as pattern for the DIC algorithm, as shown in Fig. 8(a). Later, we found that sometimes this edge contrast was not high enough in the red and blue frames, therefore, a Pt deposition pattern in these frames was added, and the two blue frames were replaced by one blue frame at the beginning of the gauge section, see Fig. 8(b). Fine-scale Pt patterns have been used extensively in the literature for DIC on SEM images [39, 40]. Here, however, the Pt pattern has been optimized (in terms of speckle size and contrast) to yield the maximum displacement resolution for DIC applied to optical profilometry images, which explains the choice for a much coarser pattern with smooth edges of the speckles. This reduces the interpolation error in the DIC algorithm and makes the DIC more robust against out of plane displacement [41-43]. Usually, such a smooth speckle pattern is hard to make, but in our case the ion-beam assisted Pt deposition gives full control to create speckles with smooth edges. Note, however, that such a profilometry-DIC pattern may appear coarse and out-offocus when viewed under optical microscopy.

The difference between the displacement of the red frame and the blue frames is the elongation of the specimen gauge part, from which the global strain is calculated. The displacement reproducibility over the test duration of $\sim 500 \mathrm{~s}$ (Fig.9(b)) is estimated to be $\sim 10 \mathrm{~nm}$. This corresponds to a reproducible global strain resolution of $\sim 0.1 \%$, which is approximately an order of magnitude better than the typical global strain resolution found in pillar compression tests, see, e.g., the review paper of Ref. [3]. This high reproducibility is achieved because only the displacement of the boxes is correlated, i.e. the number of degrees of freedom in the GDIC algorithm is kept as low as possible. Note that the displacement resolution can be further improved considerably by conducting the experiment under in-situ SEM observation with higher magnification with much more pixels in the field of view and using a much finer Pt deposition pattern; in that case, the resolution is expected to be limited by SEM scanning artifacts and drift, but this has not been tested. In addition to the red and blue frames, 


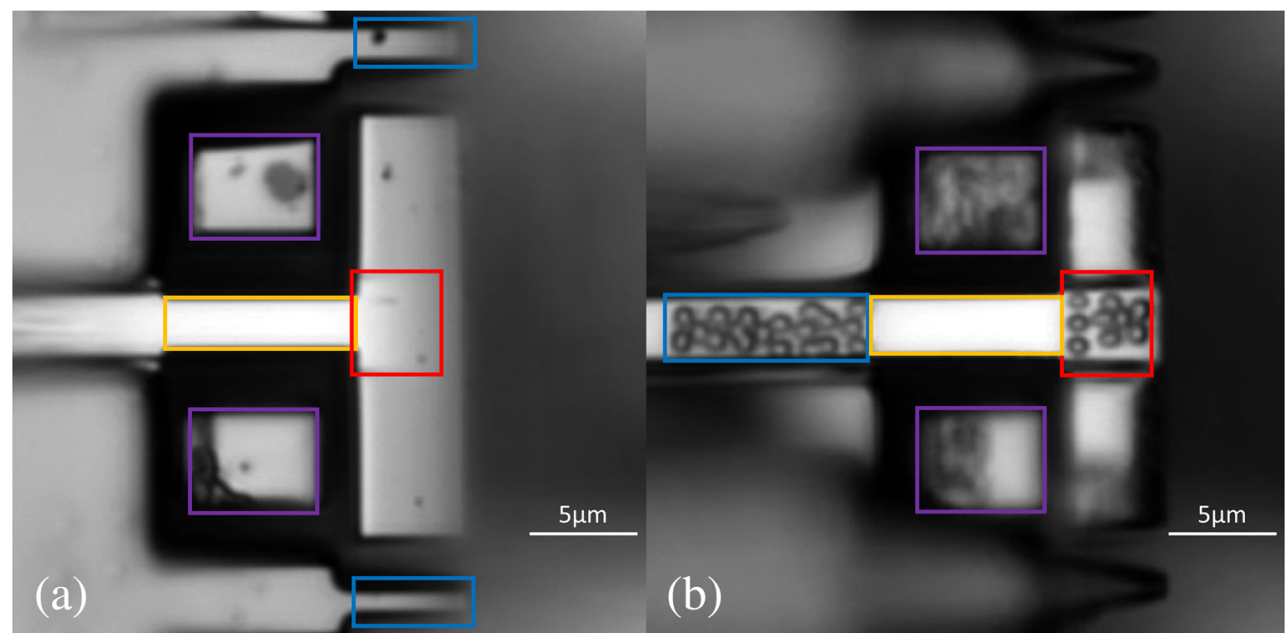

Fig. 8 Displacement measurement using global digital image correlation, using natural patterns on the specimen (a) and Pt-deposited patterns (b) [44, 45]. The total elongation of the specimen gauge (yellow frame) is obtained from the difference in rigid body displacement between the red and blue frame. The purple frames are used to track the displacement of the gripper teeth in order to determine the starting point of loading (Fig. 9(b)). FIB-assisted Pt-deposition has been used to create the dots that serve as DIC tracking pattern; note that this is only needed when the specimens do not have a natural pattern. Note that in Fig. 9(b) only the specimen gauge section is in focus because of the shallow depth of field of optical microscope at high magnifications

the purple frames that contain the teeth of the grippers are also traced, in order to determine the precise touching point of the gripper to the specimen, which is the starting time of loading (Fig.9(b)). Finally, the proposed experimental methodology is designed and ready for in-situ testing under SEM imaging, therefore, with addition of a Pt pattern on the gauge section, an in-situ SEM-DIC analysis of the full deformation field of the specimen is a measurement possibility (not shown here).

The micro-tensile test is initiated by pulling the crossbar to the micro-specimen with the gripper teeth, which leads to a steady increase of the force on the force-time curve (Fig. 9(a)). On the gripper displacement-time curve (Fig. 9(b)), the (initially high) slope of the curve reflects the displacement rate of the freely moving gripper which follows the applied displacement rate of the leaf spring mechanism. After the gripper makes contact with the specimen crossbar, the gripper displacement rate significantely decreases, which is accommodated by deflection of the leaf spring. This load initiation point is indicated by the arrows in Figs. 9(a,b). The force and displacement data can be accurately synchronized by aligning the load initiation points in time, which are determined by fitting two lines to each curve. An example of the resulting engineering stress-strain curve is shown in Fig. 9(c). The apparent irregularities in the plastic part of the stress-strain curve are caused by the inhomogeneous deformation in the gauge section of the micro-specimen.

\section{Proof of Principle}

\section{Case 1: Single Phase Specimens}

As a proof of principle, a set of specimens is fabricated to verify the reliability and accuracy of the test methodology. Fig. 10(a) shows three tensile specimens from the same grain on a wedge produced from bulk IF steel. The material was

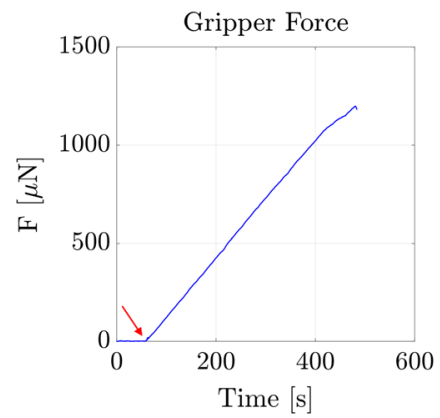

(a)

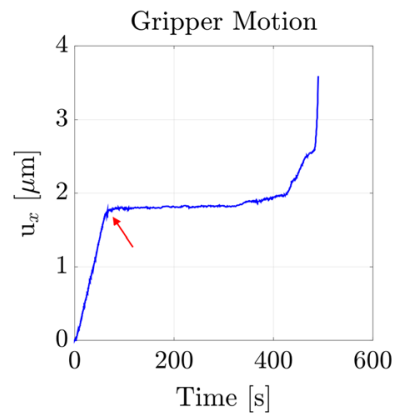

(b)

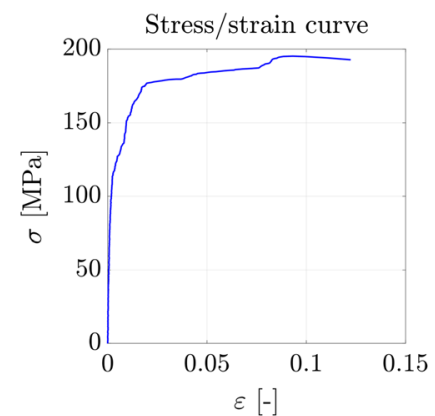

(c)

Fig. 9 (a) Force-time curve and (b) displacement-time curve of the gripper. The points marked by arrows are the transition points in the curves, which indicates the starting moment of loading. (c) Global engineering stress-strain curve obtained by combining of (a, b). The noise level of the force measurement, corrected for background influences, is considerably smaller than the applied load in the micro-tensile specimens 

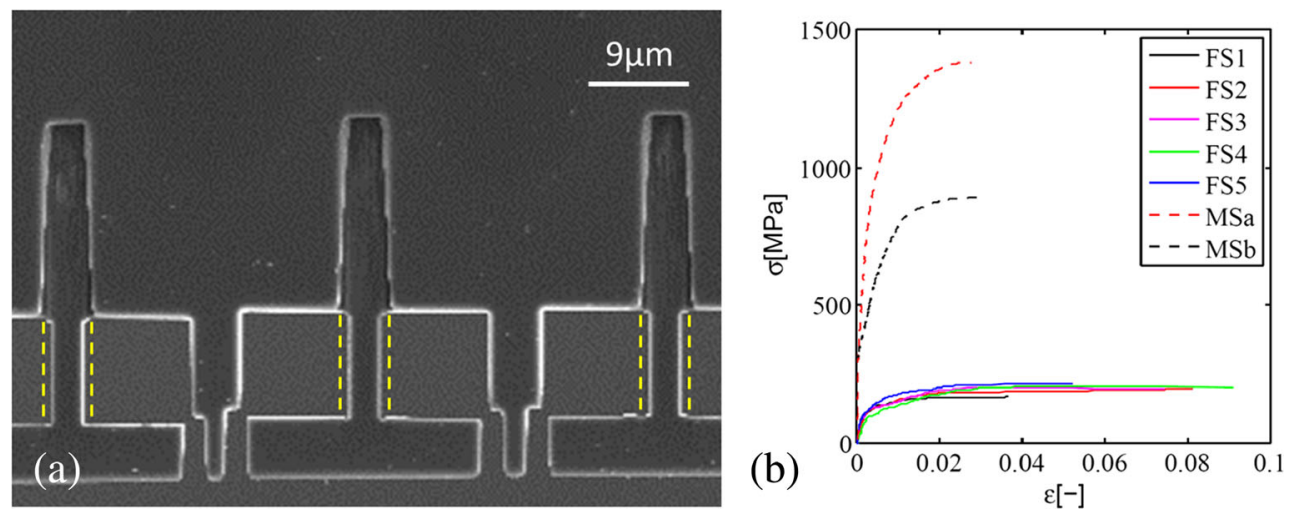

Fig. 10 An example of 5 parallel micro-specimens from a single ferrite grain. (a) Zoom of 3 specimens, where yellow dashed lines mark the high level of parallelism between the micro-specimens. (b) Engineering stress-strain curves of the 5 ferrite micro-specimens, shown together with the engineering stress-strain curves of the 2 martensite specimens discussed below. The initial length of all specimens is $9 \mu \mathrm{m}$. Note that due to the high deformation speed close to fracture, the actual fracture strain may be larger than calculated with GDIC from the last captured image

heat treated to increase the grain size such that multiple microtensile specimens could be fabricated from a single grain. The high level of parallelism among the tensile specimens reduces the amount of alignment work to only align the first specimen in the series.

The flow curves of the ferrite specimens are shown in Fig. 10(b). It is well known from the literature that the variability in the flow curves can be large for micron-sized specimens (even larger than shown here) [3, 46, 47]. This variability between flow curves is due to large local fluctuations in the density of dislocations, alloying elements and nano-carbides at this small scale. The different plateaus in the flow curves can be due to the different hardening resulted from different interaction of slip systems. However, a detailed slip trace analysis is needed to investigate this further.

To this end, SE and BSE images of the specimens after deformation are shown in Fig. 11(a-e). The slip systems are marked and identified by the traces on the specimen surfaces. Note that the methodology allows for inspection of all sides of the specimen, as shown in Fig. 11(f), to enable a clear identification of slip planes, which is especially useful for cases where the surface traces of different slip systems overlap on the front surface of the specimens. All 5 specimens show the same active slip systems, which demonstrates the overall reproducibility of the methodology for the applied loading, specimen alignment, and micro-specimen geometry. The observed surface traces of dislocations match well with the theoretical ones, both on the FIBmilled side and the FIB-free side of the specimen. This suggests that the influence of FIB milling on the failure behavior of specimens is negligible. Nevertheless, some influence of FIB milling on the plasticity and fracture behavior cannot be excluded. The slip system activation mechanism influenced by the boundary constraint is revealed by this method. This mechanism is hard to observe with macro-mechanical testing because the observed behavior of macro-specimens is the averaged behavior of huge number of grains. It is also difficult to observe by other micro-mechanical testing methods which have more complicated stress-state of the specimens.

Of course, it is not a real challenge to isolate a single-crystal specimen from a large-grained bulk material. Yet, the small dimension of the wedge edge used in the current methodology allows for fabrication of singlecrystal micro-specimens from multiphase materials with a realistic microstructure. In Fig. 12(a), an example of a

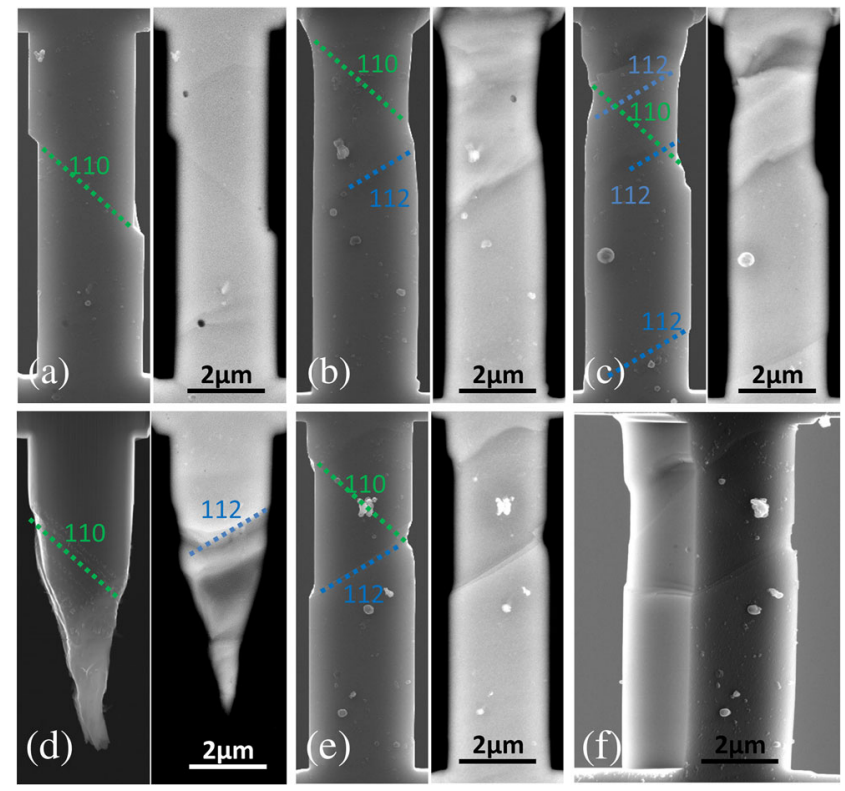

Fig. 11 (a-e) SE and backscattered electron (BSE) SEM images of the front side of $5 \mu$-specimens taken from a single ferrite grain. The main slip traces from the $\{110\}<111>$ and $\{112\}<111>$ slip families are indicated in the SE images. (f) The methodology allows for inspection of all sides of the micro-specimens to enable a clean identification of slip planes, as demonstrated here by a tilted side view of specimen (e). The initial length of the specimens is $9 \mu \mathrm{m}$ 

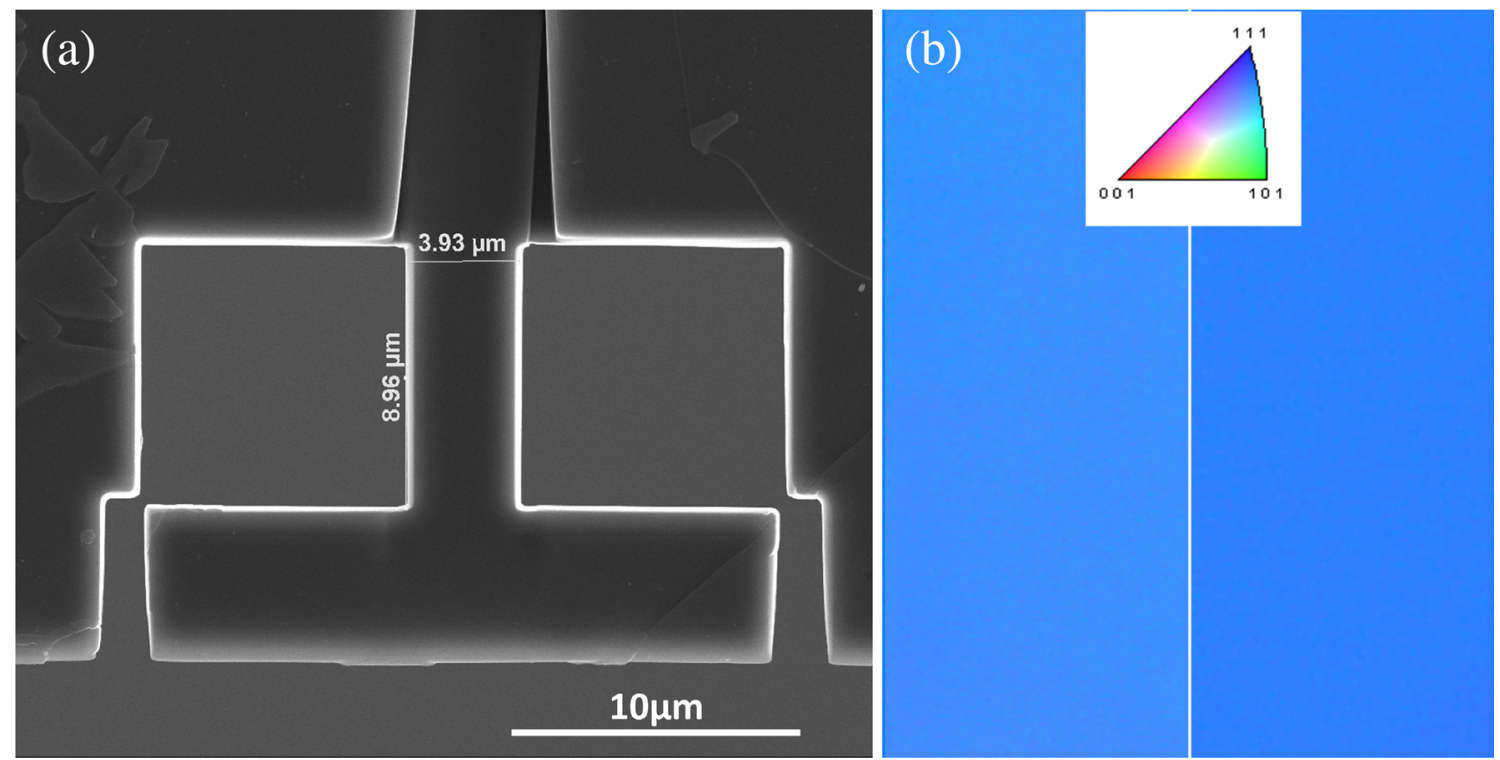

Fig. 12 (a) SEM micrograph of a ferrite micro-specimen extracted from a DP steel. Martensite islands are visible outside the gauge section. (b) EBSD inverse pole figure map of the front side and back side of the specimen gauge part, which reveals its single crystal nature

ferrite micro-specimen isolated from a dual phase steel sheet is shown, where the martensite islands are still visible, e.g. on the top left of the image. As shown by the inverse pole figure in Fig. 12(b), the front and back side shows the same inverse pole figure, which confirms that the specimen gauge part contains only a single ferrite grain, extracted from a multiphase material.

\section{Case 2: Micro-Specimens Based on Crystallographic Orientation Selection}

This case illustrates lath martensite specimen preparation based on orientation selection. A so-called packet is composed of parallel stacks of laths, which is the fundamental structure unit of lath martensite. These laths can be grouped into sub-blocks and blocks which have a width of micrometers based on the misorientation between them. Therefore, the proposed methodology allows to reveal the effect of the sub-block and block boundaries on the strength of lath martensite.

To obtain the correct boundary configuration in the specimen gauge part, it is required to fabricate specimens from a site-specific region of martensite based on prior EBSD analysis. In the ideal case, a constant boundary through the thickness of the specimen gauge section is preferred, which means inclinations of boundaries in $3 \mathrm{D}$ within the specimen are demanded. The selection of the specimen fabrication sites can be based on (1) the pole figures obtained by the EBSD measurement (The martensite lath boundary is approximately parallel to the $\{110\}$ planes. Therefore, the $\{110\}$ pole figures of martensite can be used to judge if the boundary is perpendicular to the front plane of the specimen.) and (2) the evaluation of the EBSD inverse pole figure maps from the front and the back sides of the specimen gauge part.

Two configurations of boundary orientations are shown, where (1) the lath boundary is perpendicular to the surface of the specimen, and also parallel to the loading direction (Fig. 13(a)) and (2) the lath boundary is perpendicular to the surface of the specimen, but with an angle of $45^{\circ}$ to the loading direction (Fig. 13(b)). The projection points marked in red circles on the periphery of the equatorial plane of the pole figures (Fig. 13(a5, b5)) indicate that the corresponding (110) plane is constant in the thickness direction of the specimen gauge part. A confirmation of this observation is given by the EBSD map of the backside of the specimens in Fig. 13(a3,b3). The SEM images of the two specimens before and after deformation are shown in Fig. 13(a1,4) and Fig. 13(b1,4) respectively. The engineering stress-strain curves of these two specimens are given in Fig. 10(b), which clearly show the difference of stress levels between the two deformation mechanisms (and the fact that martensite is obviously much stronger than ferrite).

Based on these micro-tensile tests, two new micromechanisms of lath martensite are demonstrated: (1) in contrast to earlier reports in the literature relying on indirect measurements, sub-block boundary strengthening is (almost) equally important as block boundary strengthening [44], and (2) under favorable orientation, all lath martensite sub-structure boundaries can exhibit boundary sliding, which competes with crystallographic slips as the primary deformation mechanism [45]. The identification of 
Fig. 13 Examples of selection of micro-specimens based on orientations of the laths. The SEM images of the specimens before deformation (a1, b1) and after deformation $(\mathrm{a} 4, \mathrm{~b} 4)$ of the front side are presented. The lath boundary of specimen a is parallel to the loading direction, which is the vertical direction here, whereas the lath boundaries of specimen $\mathrm{b}$ is roughly $45^{\circ}$ tilted with respect to the loading direction. Due to the fact that the boundaries are roughly parallel to the $\{110\}$ planes of the lath, the projection points in the read circles at the periphery in the $\{110\}$ pole figure $(\mathrm{a} 5, \mathrm{~b} 5)$ indicate that the boundary plane of the laths are perpendicular to the specimen surface. This can be confirmed by the EBSD map of the front side and backside of the gauge sections shown in $(\mathrm{a} 2, \mathrm{a} 3)$ and $(\mathrm{b} 2, \mathrm{~b} 3)$. Note that the backside EBSD maps are flipped horizontally. The engineering stress-strain curves of these two specimens are shown in Fig. 10(b), marked as MSa and MSb respectively. The initial length of the specimens is $9 \mu \mathrm{m}$
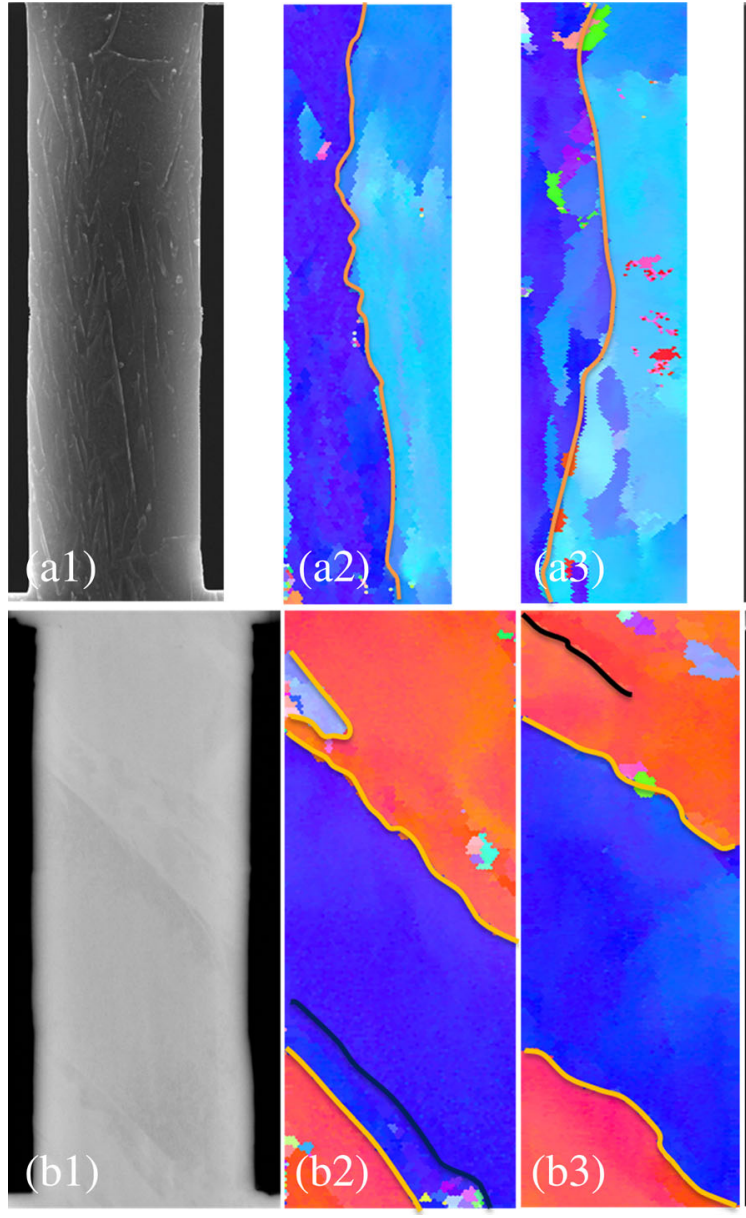
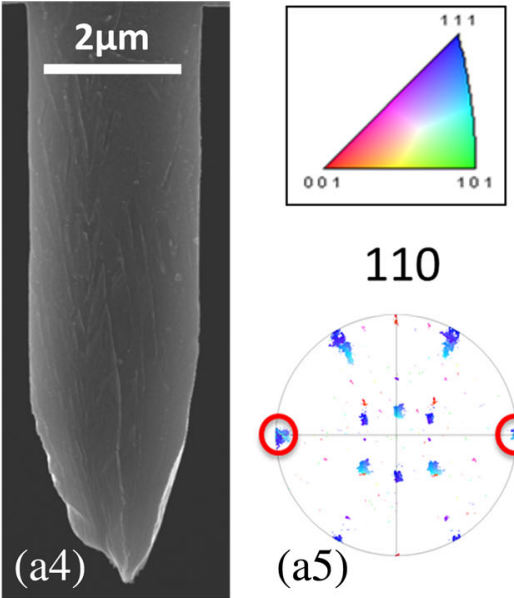

110
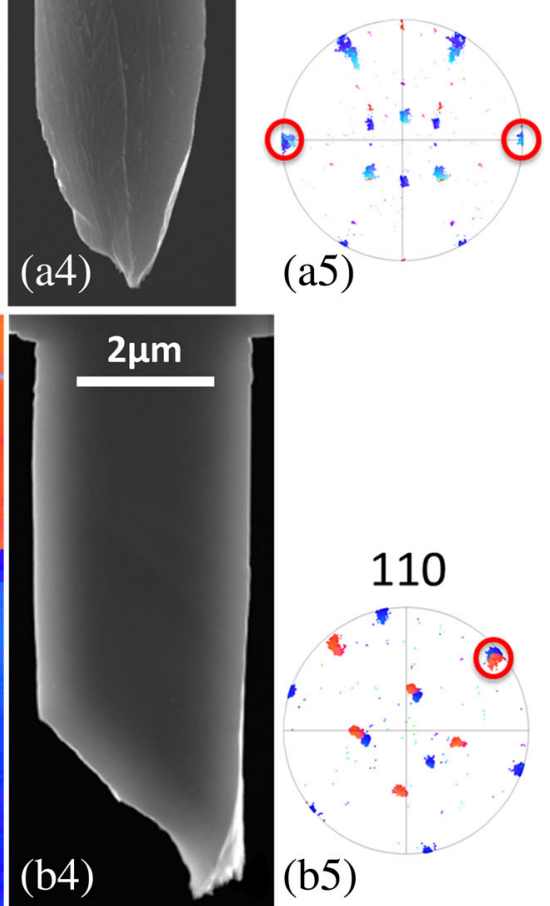

these micro-mechanisms is only possible because the used methodology allows for crystallographic orientation specimen selection, combined with front and backside EBSD mapping of the microstructure.

\section{Conclusions}

A reliable and reproducible micro-tensile test is proposed, designed and demonstrated with examples of single phase and multiphase materials together with region selection based on EBSD measurement. We have shown that:

(1) The wedge concept allows fabrication of multiple parallel micro-tensile specimens from a single wedge. With this concept, it is easy to handle micro-tensile specimens and the amount of alignment work is significantly reduced.

(2) A site-specific specimen fabrication method is established for micro-tensile specimen preparation from bulk materials, which is applicable to both single-phase materials and multiphase materials with possibility to select regions for specimens based on crystallographic orientations or morphologies.

(3) A setup for micro-tensile testing is constructed with accurate specimen alignment, force and displacement measurement. The setup is adapted for in-situ testing to obtain a clean observation of the specimen surfaces in the complete experiment.

(4) This testing setup is able to reveal mechanisms that are difficult to identify with macro-mechanical testing. Due to the simple loading condition, the analysis of observations is more straightforward compared to other micromechanical tests with more complex loading conditions.

Acknowledgements This research was carried out under project number M22.2.11424 in the framework of the Research Program of the Materials innovation institute (M2i) (www.m2i.nl).

Open Access This article is distributed under the terms of the Creative Commons Attribution 4.0 International License (http:// creativecommons.org/licenses/by/4.0/), which permits unrestricted use, distribution, and reproduction in any medium, provided you give appropriate credit to the original author(s) and the source, provide a link to the Creative Commons license, and indicate if changes were made. 


\section{References}

1. Santofimia MJ, Kwakernaak C, Sloof WG, Zhao L, Sietsma J (2010) Experimental study of the distribution of alloying elements after the formation of epitaxial ferrite upon cooling in a low-carbon steel. Mater Charact 61:937-942

2. Franciosi P, Le LT, Monnet G, Kahloun C, Chavanne MH (2015) Investigation of slip system activity in iron at room temperature by SEM and AFM in-situ tensile and compression tests of iron single crystals. Int J Plasticity 65:226-249

3. Greer JR, De Hosson JTM (2011) Plasticity in small-sized metallic systems: intrinsic versus extrinsic size effect. Prog Mater Sci 56: 654-724

4. Kuzmin OV, Pei YT, Chen CQ, De Hosson JTM (2012) Intrinsic and extrinsic size effects in the deformation of metallic glass nanopillars. Aata Mater 60:889-898

5. Krauss G (1999) Martensite in steel: strength and structure. Mat Sci Eng A-Struct 273-275:40-57

6. Morito S, Yoshida H, Maki T, Huang X (2006) Effect of block size on the strength of lath martensite in low carbon steels. Mat Sci Eng A-Struct 438-440:237-240

7. Zhang C, Wang Q, Ren J, Li R, Wang M, Zhang F, Sun K (2012) Effect of martensitic morphology on mechanical properties of an asquenched and tempered 25CrMo48V steel. Mat Sci Eng A-Struct 534:339-346

8. Shibata A, Nagoshi T, Sone M, Morito S, Higo Y (2010) Evaluation of the block boundary and sub-block boundary strengths of ferrous lath martensite using a micro-bending test. Mat Sci Eng A-Struct 527:7538-7544

9. Shibata A, Nagoshi T, Sone M, Morito S, Higo Y (2013) Micromechanical characterization of deformation behavior in ferrous lath martensite. J. Alloys Compd 577:S555-S558

10. Liu D, He Y, Tang X, Ding H, Hu P, Cao P (2012) Size effects in the torsion of microscale copper wires: experiment and analysis. Scripta Mater 66:406-409

11. Delince M, Jacques PJ, Pardoen T (2006) Separation of sizedependent strengthening contributions in fine-grained dual phase steels by nanoindentation. Acta Mater 54:3395-3404

12. Tasan CC, Hoefnagels JPM, Geers MGD (2009) A critical assessment of indentation-based ductile damage quantification. Acta Mater 57:4957-4966

13. Jirkova H, Masek B, Wagner MFX, Langmajerova D, Kucerova L, Treml R, Kiener D (2014) Influence of metastable retained austenite on macro and micromechanical properties of steel processed by the Q\&P process. J. Alloys Compd 615:S163-S168

14. Kheradmand N, Vehoff H, Barnoush A (2013) An insight into the role of the grain boundary in plastic deformation by means of a bicrystalline pillar compression test and atomistic simulation. Acta Mater 61:7454-7465

15. Duesbery MS, Vitek V (1998) Plastic anisotropy in b.C.c. Transition metals. Acta Mater 46:1481-1492

16. Uchic MD, Dimiduk DM, Florando JN, Nix WD (2004) Sample dimensions' influence strength and crystal plasticity. Science 305: 986-989

17. Uchic MD, Dimiduk DM (2005) A methodology to investigate size scale effects in crystalline plasticity using uniaxial compression testing. Mat Sci Eng A-Struct 400-401:268-278

18. Motz C, Schoeberl T, Pippan R (2005) Mechanical properties of micro-sized copper bending beams machined by the focused ion beam technique. Acta Mater 53:4269-4279

19. Guo H, Yan PF, Wang YB, Tan J, Zhang ZF, Sui ML, Ma E (2007) Tensile ductility and necking of metallic glass. Nat Mater 6:735739

20. Kuzmin OV, Pei YT, De Hosson JTM (2014) Nanopillar fabrication with focused ion beam milling. Microsc Microanal 20:1581-1584
21. Kang W, Han JH, Saif MTA (2010) A novel method for in situ uniaxial tests at micro/nanoscale-part 2: experiment. J Microelectromech Syst 19:1322-1330

22. Kiener D, Grosinger W, Dehm G, Pippan R (2008) A further step towards an understanding of size-dependent crystal plasticity: in situ tension experiments of miniaturized single-crystal copper specimens. Acta Mater 56:580-592

23. Yu J, Liu J, Zhang J, Wu J (2006) TEM investigation of FIB induced damages in preparation of metal material TEM specimens by FIB. Mater Lett 60:206-209

24. Ando M, Tanigawa H, Jitsukawa S, Sawai T, Katoh Y, Kohyama A, Nakamura K, Takeuchi H (2002) Evaluation of hardening behavior of ion irradiated reduced activation ferritic/martensitic steel by an ultra-micro-indentation technique. J Nucl Mater 307-311:260-265

25. McCaffrey JP, Phaneuf MW, Madsen LD (2001) Surface damage formation during ion-beam thinning of samples for transmission electron microscopy. Ultramicroscopy 87:97-104

26. Giannuzzi LA, Stevie FA (1999) A review of focused ion beam milling techniques for TEM specimen preparation. Micron 30: 197-204

27. Kiener D, Motz C, Rester M, Jenko M, Dehm G (2007) FIB damage of cu and possible consequences for miniaturized mechanical tests. Mat Sci Eng A-Struct 459:262-272

28. Babu PR, Irukuvarghula S, Harte A, Preuss M (2016) Nature of gallium focused ion beam induced phase transformation in 316 austenitic stainless steel. Acta Mater 120:391-402

29. Unocic KA, Mills MJ, Daehn GS (2010) Effect of gallium focused ion beam milling on preparation of aluminum thin foils. J Microsc 240:227-238

30. Jennings AT, Burek MJ, Greer JR (2010) Microstructure versus size: mechanical properties of electroplated single crystalline $\mathrm{cu}$ nanopillars. Phys Rev Lett 104:135503

31. Zimmermann J, Van Petegem S, Bei H, Grolimund D, George EP, Van Swygenhoven H (2010) Effects of focused ion beam milling and pre-straining on the microstructure of directionally solidified molybdenum pillars: a Laue diffraction analysis. Scrip Mater 62: 746-739

32. Zaefferer $\mathrm{S}$ (2007) On the formation mechanisms, spatial resolution and intensity of backscatter Kikuchi patterns. Ultramicroscopy 107: 254-266

33. Uchic DM, Shade PA, Dimiduk DM (2009) Plasticity of micrometer-scale single crystals in compression. Annu Rev Mater Res 39:361-386

34. Guo EY, Xie HX, Singh SS, Kirubanandham A, Jing T, Chawla N (2014) Mechanical characterization of microconstituents in a cast duplex stainless steel by micropillar compression. Mat Sci Eng AStruct 598:98-105

35. Bergers LIJC, Hoefnagels JPM, Geers MGD (2014) On-wafer time-dependent high reproducibility nano-force tensile testing. J Phys D Appl Phys 47:495306

36. Bergers LIJC, Hoefnagels JPM, Geers MGD (2014) Characterization of time-dependent anelastic microbeam bending mechanics. J Phys D Appl Phys 47:355306

37. Neggers J, Hoefnagels JPM, Hild F, Roux S, Geers MGD (2014) Direct stress-strain measurements from bulged membranes using topography image correlation. Exp Mech 54: 717-727

38. Naraghi M, Chasiotis I, Kahn H, Wen Y, Dzenis Y (2007) Novel method for mechanical characterization of polymeric nanofibers. Review Sci Instrum 78:085108

39. Bemporad E, Brisotto M, Depero LE, Gelfi M, Korsunsky AM, Lunt AJG, Sebastiani M (2014) A critical comparison between XRD and FIB residual stress measurement techniques in thin films. Thin Solid Films 572:224-231 
40. Winiarski B, Schajer GS, Withers PJ (2012) Surface decoration of improving the accuracy of displacement measurements by digital image correlation in SEM. Exp Mech 52:793-804

41. Sun Y, Pang JHL (2007) Study of optimal subset size in digital image correlation of speckle pattern images. Opt Laser Eng 45: 967-974

42. Pan B (2013) Bias Error reduction of digital image correlation using Gaussian pre-filtering. Opt Laser Eng 51:1161-1167

43. Ruybalid AP, Hoefnagels JPM, Van der Sluis O, Geers MGD (2016) Comparison of the identification performance of conventional FEM-updating and integrated DIC. Int J Numer Methods Eng 106:298-320
44. Du C, Hoefnagels JPM, Vaes R, Geers MGD (2016) Block and subblock boundary strengthening in lath martensite. Scripta Mater 116: $117-121$

45. Du C, Hoefnagels JPM, Vaes R, Geers MGD (2016) Plasticity of lath martensite by sliding of substructure boundaries. Scripta Mater $120: 37-40$

46. Greer JR, Oliver WC, Nix WD (2005) Size dependence of mechanical properties of gold at the micron scale in the absence of strain gradients. Acta Mater 53:1821-1830

47. Ghassemi-Armaki H, Maass R, Bhat SP, Sriram S, Greer JR, Kumar KS (2014) Deformation response of ferrite and martensite in a dualphase steel. Acta Mater 62:197-211 